\title{
Gemini Planet Imager observational calibrations XV: instrument calibrations after six years on sky
}

De Rosa, Robert, Esposito, Thomas, Gibbs, Aidan, Bailey, Vanessa, Fitzgerald, Michael, et al.

Robert J. De Rosa, Thomas M. Esposito, Aidan Gibbs, Vanessa P. Bailey, Michael P. Fitzgerald, Jeffrey Chilcote, Gaspard Duchêne, Quinn Konopacky, Bruce Macintosh, Maxwell A. Millar-Blanchaer, Meiji M. Nguyen, Eric L. Nielsen, Marshall D. Perrin, Julien Rameau, Jason J. Wang, "Gemini Planet Imager observational calibrations XV: instrument calibrations after six years on sky," Proc. SPIE 11447, Ground-based and Airborne Instrumentation for Astronomy VIII, 114475A (13 December 2020); doi: 10.1117/12.2561071 


\title{
Gemini Planet Imager Observational Calibrations XV: Instrument calibrations after six years on sky
}

Robert J. De Rosa ${ }^{\mathrm{a}}$, Thomas M. Esposito ${ }^{\mathrm{b}, \mathrm{c}}$, Aidan Gibbs ${ }^{\mathrm{d}}$, Vanessa P. Bailey ${ }^{\mathrm{e}}$, Michael P. Fitzgerald $^{\mathrm{d}}$, Jeffrey Chilcote ${ }^{\mathrm{f}}$, Gaspard Duchêne ${ }^{\mathrm{b}, \mathrm{g}}$, Quinn Konopacky ${ }^{\mathrm{h}}$, Bruce Macintosh ${ }^{\mathrm{i}}$, Maxwell A. Millar-Blanchaer ${ }^{\mathrm{j}}$, Meiji M. Nguyen ${ }^{\mathrm{b}}$, Eric L. Nielsen ${ }^{\mathrm{k}}$, Marshall D. Perrin ${ }^{1}$, Julien Rameau ${ }^{\mathrm{g}, \mathrm{m}}$, and Jason J. Wang ${ }^{\mathrm{n}}$

${ }^{a}$ European Southern Observatory, Alonso de Córdova 3107, Vitacura, Santiago, Chile

${ }^{\mathrm{b}}$ Department of Astronomy, University of California, Berkeley, CA 94720, USA

'SETI Institute, Carl Sagan Center, 189 Bernardo Avenue, Mountain View CA 94043, USA

${ }^{\mathrm{d}}$ Department of Physics \& Astronomy, 430 Portola Plaza, University of California, Los Angeles, CA 90095, USA

e Jet Propulsion Laboratory, California Institute of Technology, Pasadena, CA 91109, USA

${ }^{\mathrm{f}}$ Department of Physics, University of Notre Dame, 225 Nieuwland Science Hall, Notre Dame, IN 46556, USA

${ }^{\mathrm{g} U n i v . ~ G r e n o b l e ~ A l p e s / C N R S, ~ I P A G, ~ F-38000 ~ G r e n o b l e, ~ F r a n c e ~}$

${ }^{\mathrm{h}}$ Center for Astrophysics and Space Science, University of California San Diego, La Jolla, CA 92093, USA

${ }^{\mathrm{i}}$ Kavli Institute for Particle Astrophysics and Cosmology, Stanford University, Stanford, CA 94305, USA

${ }^{j}$ Department of Physics, University of California, Santa Barbara, Santa Barbara, CA 93106, USA

${ }^{k}$ Department of Astronomy, New Mexico State University, 1320 Frenger Mall, Las Cruces, NM 88003, USA

${ }^{1}$ Space Telescope Science Institute, Baltimore, MD 21218, USA

minstitut de Recherche sur les Exoplanètes, Département de Physique, Université de Montréal, Montréal QC, H3C 3J7, Canada

${ }^{\mathrm{n}}$ Department of Astronomy, California Institute of Technology, Pasadena, CA 91125, USA

\begin{abstract}
The Gemini Planet Imager (GPI) is a high-contrast adaptive optics instrument designed to detect and characterize substellar companions and circumstellar debris disks around nearby young stars using infrared integral field spectroscopy and polarimetry. GPI has been in routine operations at Gemini South for the past six years and will be transferred to Gemini North in the coming years after an upgrade of several of its components. Because precise astrometry and photometry of exoplanets is critical to GPI's science, we undertook extensive efforts both in-lab and on-sky to refine the astrometric and photometric calibration of the instrument. Here we describe revisions to the GPI Data Reduction Pipeline (DRP) that account for these revised calibrations, and that fix several issues identified over the previous six years, including some subtle issues affecting astrometric calibrations caused by a drift of the instrument's clock. These calibrations are critical for the interpretation of observations obtained with GPI, and for a comparison with measurements from other high-contrast imaging instruments.
\end{abstract}

Keywords: Keywords

E-mail: rderosa@eso.org

Ground-based and Airborne Instrumentation for Astronomy VIII, edited by Christopher J. Evans,

Julia J. Bryant, Kentaro Motohara, Proc. of SPIE Vol. 11447, 114475A · @ 2020

SPIE - CCC code: $0277-786 X / 20 / \$ 21 \cdot$ doi: $10.1117 / 12.2561071$ 


\section{INTRODUCTION}

The Gemini Planet Imager ${ }^{1}$ (GPI) is a facility instrument of the 8-m Gemini South telescope on Cerro Pachon, Chile. The instrument combines a high-order adaptive optics (AO) system ${ }^{2}$ with an apodized Lyot coronagraph ${ }^{3}$ allowing for high Strehl ratio, ${ }^{4,5}$ high-contrast ${ }^{6}$ observations of the immediate vicinity of nearby, bright stars. Behind the AO system and coronagraph lies a lenslet-based integral field spectrograph ${ }^{7,8}$ (IFS) covering a field of view of approximately $2.8 \times 2.8$ arcseconds. The corrected beam can either pass through a low-resolution dispersing prism for integral field spectroscopy, or through a Wollaston prism for integral field polarimetry. ${ }^{9}$ These various components gave GPI unparalleled sensitivity to the faint thermal emission from massive gas giant exoplanets, as well as the polarized scattered-light emission from large circumstellar debris disks, around nearby young stars.

Since early-2014 GPI has been used to detect the most Jupiter-like exoplanet directly imaged, ${ }^{10,11}$ to place the best constraints on the frequency and distribution of wide-orbit planets, ${ }^{12}$ and to study the morphology and composition of a wide array of circumstellar debris disks. ${ }^{13}$ These studies all rely on having precise and accurate instrumental calibrations in order to convert the images recorded on the detector into a well-calibrated data product such as a polarized-intensity image of a debris disk or a spectrum of a substellar companion. An extensive effort ${ }^{14}$ was made before and during the commissioning of the instrument to provide the user community with the calibrations necessary to interpret their observations. These were based on a combination of experiments conducted while the instrument was being built and measurements obtained during commissioning either on-sky or using GPI's internal Artificial Star Unit (ASU).

Despite the commissioning activities concluding in mid2014, we have continued to monitor several aspects of the instrument calibration. In particular, the astrometric and photometric calibration of the instrument was revisited given the importance of these calibrations on the interpretation of observations of substellar companions, and their comparison with data obtained using other high-contrast imaging instruments (e.g., SPHERE ${ }^{16}$ ). We have also revised the data reduction pipeline for data obtained with the Wollaston prism to better subtract the instrumental polarization noise present in polarimetric images of circumstellar debris disks. Here we report on revisions to these calibrations based on data obtained during the past six years of instrument operations.

\section{SATELLITE SPOT CALIBRATION}

\subsection{Overview}

High-contrast imaging with a coronagraph makes it impossible to measure directly the position and brightness of the star being targeted. This makes it challenging to perform precision relative astrometry and photometry between the target star and a resolved companion. To overcome this, GPI uses ${ }^{17,18}$ a wire diffraction grating imprinted on the
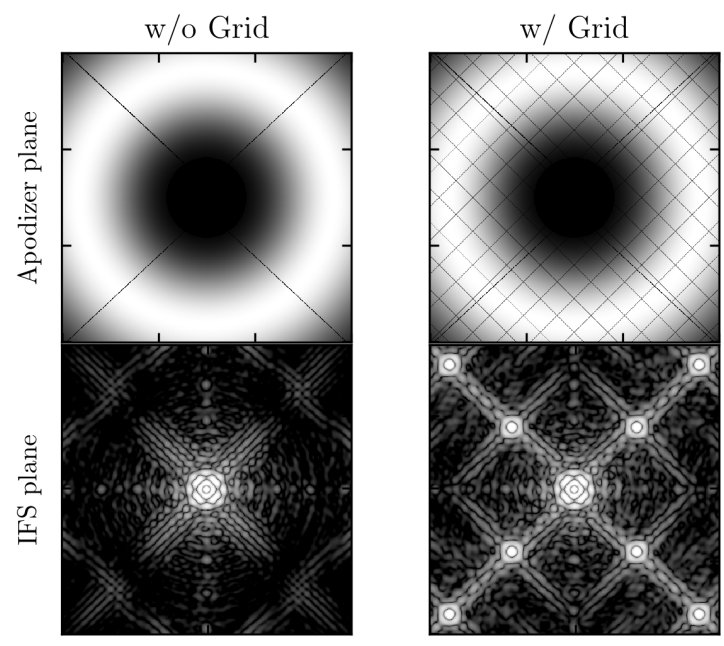

Figure 1. Pupil-plane image at the location of the apodizer (top row) and log-scaled focal-plane image at the IFS (bottom row) with (left column) and without (right column) the grid imprinted on the apodizer, simulated using the poppy package. ${ }^{15}$ The width of the grid lines sets the flux ratio, and the spacing between the grid lines sets the position in the focal plane. pupil plane apodizer that generates attenuated replicas of the central source in a periodic grid within the focal plane ${ }^{19}$ (Fig. 1). These replicas, or "satellite spots", can be used to determine the location of the star and, if calibrated, its brightness. The separation between the central point spread function (PSF) and the satellite spots is governed by the spacing of the grid lines in the pupil plane, and the ratio of their fluxes is governed by the width of the grid lines. For GPI, the grids imprinted on the apodizers were designed to position the satellite spots at a separation of $\sim 20 \lambda / D$ with a flux ratio of $\sim 10^{-4}$. Each IFS filter was paired with an apodizer optimized for the central wavelength of the filter, except for the two $K$ band 


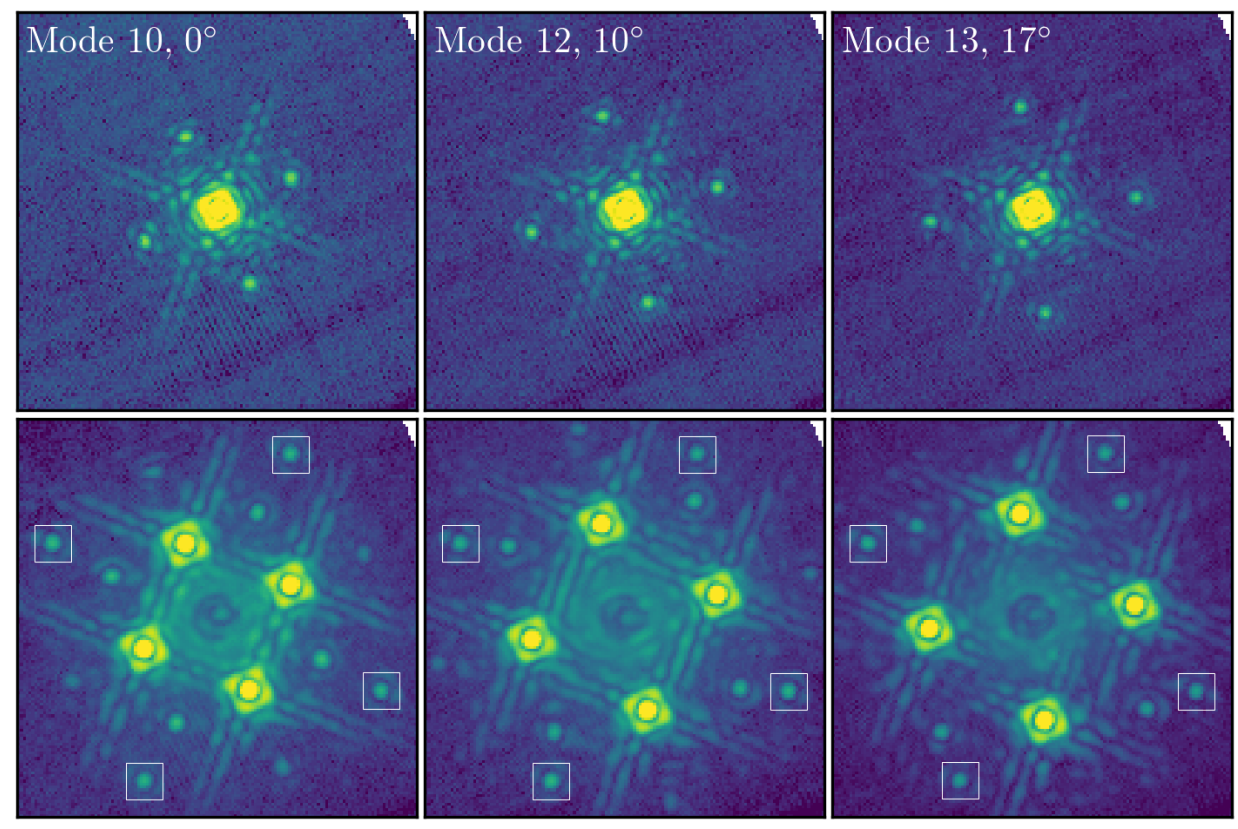

Figure 2. DM spots for three different configurations of the sine wave applied to the MEMS as seen in the ND images (top row) and coronagraphic images (bottom row). The mode corresponds to the spatial frequency of the sine wave (larger mode, higher frequency, spots at a larger separation), and the angle corresponds to the counter-clockwise rotation of the spots in the IFS image. The location of the satellite spots generated by the apodizer grating are highlighted by boxes, not to be confused with the additional sources generated by the bright DM spots.

filters that used the same apodizer. The diffraction grating also creates higher order spots at integer multiples of the $\sim 20 \lambda / D$ separation; these are visible in IFS images at shorter wavelengths ( $Y$ and $J$ band). The ratio between these second order spots and the central source was not characterized in the previous analysis. ${ }^{17}$

This revision of the satellite spot calibration was motivated by a fault in GPI's pupil plane mechanism (PPM) in 2015 that caused all observations regardless of wavelength to be taken with the apodizer optimized for the $H$-band. At the time of the previous calibration work ${ }^{17}$ it was not envisaged that the instrument would be used in a non-standard configuration where the IFS filter was not matched with the apodizer optimized for that wavelength. Due to this mechanism fault, the $H$-band apodizer was used for all observations between mid-2015 and mid-2016, regardless of which IFS filter was selected. As the flux ratio of the satellite spots in these nonstandard configurations was not previously tested the ratio was assumed to be monochromatic between 1 and $2.5 \mu \mathrm{m}$. Additional calibration measurements were required to confirm this assumption, and the opportunity was taken to confirm the original calibration values for the standard instrument modes.

\subsection{Experimental design}

The large flux ratio between the central PSF and the attenuated satellite spots prevents a direct measurement of the satellite spot ratio in a single measurement given the dynamic range of the detector. Instead, a two-step approach is used. Two perpendicular sine wave patterns of equal amplitude are applied to the MEMS deformable mirror (DM) to generate four "DM spots" at a 1:100 flux ratio to the central source, the brightness of the spots being proportional to the square of the amplitude of the sine wave. The separation of the DM spots is set by the spatial frequency, or "mode number" of the DM sine waves, and their clocking by the orientation of the sine waves on the DM. Higher mode numbers correspond to higher spatial frequencies, and thus to more widely separated DM spots. The clocking of the DM spots is chosen such that their diffraction spikes do not overlap with the fainter satellite spots. Images taken with a neutral density (ND) filter are used to measure the flux ratio between the PSF core and the DM spots, and images taken in GPI's coronagraphic mode are used to measure 
the flux ratio between the DM spots and the satellite spots. These two ratios are multiplied together to calculate the flux ratio of the satellite spots to the PSF core.

An initial experiment to repeat the original calibration measurements taken in 2013 while the instrument was being integrated and tested revealed a significant change in the morphology of the DM spots as a function of the spatial frequency and the rotation of the sine wave on the DM (Fig. 2). At the lowest spatial frequency tested two of the DM spots appeared significantly distorted relative to the central ASU and the other two DM spots. This effect was not as prominent in the 2013 data, which was taken with similar instrument and sine wave configurations, with the DM spots appearing relatively symmetric. The PSF distortions were still present at higher spatial frequencies, although less pronounced (Fig. 2).

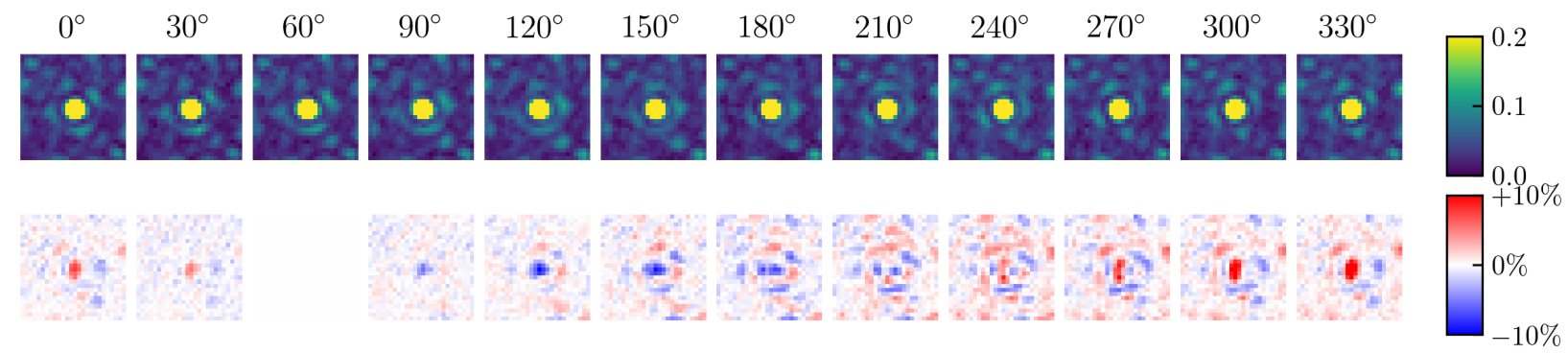

Figure 3. Effect of adjusting the phase $(\phi)$ of the sine wave applied to the MEMS on the PSF of the DM spot for 12 different phase angles (top row). The difference $\phi_{i}-\phi_{60}$ (bottom row) demonstrates the significant change in the brightness of the DM spots as a function of the phase of the applied sine wave.

It was hypothesized that the DM spots may be interfering with residual speckles from the bright central ASU ( $\sim 10^{-2}$ flux ratio), leading to a significant change in their morphology. This was confirmed in an experiment where we varied the phase of the sine wave applied to the DM from 0-360 deg (i.e. an actuator at $+1 \mu \mathrm{m}$ at 0 deg phase would be at $-1 \mu \mathrm{m}$ at $180 \mathrm{deg}$ phase). This experiment demonstrated that the brightness and morphology of the DM spots changed significantly with the phase of the sine wave (Fig. 3), with peak-to-valley amplitude variations of $40 \%$. The true flux ratio between the ASU and the average DM spot was calculated by averaging the data cubes taken at all phase angles, cancelling out the changes in the morphology induced by constructive and destructive interference. The same ratio was recovered by averaging the four images with a phase of $\phi=0$, 90, 180, and $270 \mathrm{deg}$, significantly reducing the number of phase angles required for an accurate measurement of the true flux ratio.

\subsection{Data reduction}

A typical experiment sequence consists of at least five images taken with the ND filter and five in the coronagraphic mode at each of four phase angles for the DM sine wave. Exposure times were typically between 1.5 and 6 seconds, coadded to approximately 45 seconds. There are two significant differences between these two imaging modes. 1) Images with the ND filter are taken in direct mode (no apodizer) as the ND filter is installed in the same PPM as the apodizers, and 2) for the coronagraphic images, the focal plane mechanism (FPM) is changed from SCIENCE (fully reflective) to the occulting spot corresponding to the filter (e.g., FPM_H for H-band) and the ND filter is replaced with the appropriate apodizer (e.g., APOD_H_G6205 for H-band). On dates where the satellite spot ratios for multiple apodizers with the same IFS filter were measured (e.g., J filter/J apodizer, $\mathrm{J}$ filter/H apodizer), one set of ND images were used for both sets of coronagraphic images as the required instrument configuration was identical. "Background" images were obtained for the subset of K-band datasets with the ASU off to measure the instrumental thermal background. Calibration arc and dark frames were taken when necessary.

Each dataset was reduced in a similar manner with the GPI Data Reduction Pipeline ${ }^{14}$ (DRP). The following primitives were applied to each dataset; 1) Load Wavelength Calibration, 2) Subtract Dark Background, 3) Destripe Science Image (for 2013 data only), 4) Subtract Thermal Sky Background if K Band, 5) Update Spot 
Shifts for Flexure, 6) Interpolate Bad Pixels in 2D frame, 7) Assemble Spectral Data Cube, 8) Interpolate Wavelength Axis, and 9) Interpolate Bad Pixels In Cube (for $H, K_{1}$, and $K_{2}$ datasets). Two primitives were skipped that are typically used for GPIES campaign datasets. ${ }^{20}$ The primitive "Interpolate Bad Pixels in Cube" was skipped for the shorter wavelengths $(Y, J)$ as this often caused the peaks of the DM spots to be erroneously flagged as bad pixels by the outlier rejection algorithm (although the central pixels themselves were in the linear regime). The "Correct Distortion" primitive was also skipped as it was determined to have a negligible effect on the measured satellite spot ratio. Skipping the final bad pixel correction primitive caused a significant increase in the number of bad pixels in the final data cube for the $Y$ and $J$ datasets. To help mitigate this a new hot pixel map was constructed using the 60-second dark sequence obtained on 2016-12-21. This new bad pixel map was used in the reduction of all data obtained in 2016 and 2017.

\subsection{PSF fitting}

The locations of the ASU and four DM spots in the ND images, and the location of the four DM spots and four satellite spots (and four second-order spots for $\mathrm{Y}$ and $\mathrm{J}$ band) in the coronagraphic images, were measured by fitting a two-dimensional symmetric Gaussian to a small 10-pixel stamp extracted at the estimated location with a least-squares optimizer. The full images were high-pass filtered prior to extracting the small stamp to minimize potential biases in fitting faint satellite spots near bright DM spots. The pixel coordinates of the five sources in the ND images, and the eight (or twelve) sources in the coronagraphic images, were saved for each wavelength slice of each image.

Unlike the previous analysis where the two ratios were computed by fitting a two-dimensional Gaussian, ${ }^{17}$ we instead opted to use PSF fitting. This was motivated by the non-Gaussian nature of GPI's PSF, and the fact that the ND images and coronagraphic images have different PSFs (as the latter is apodized), which may cause biases in the two-dimensional Gaussian fit that may be different for the apodized and non-apodized PSF. The ratio between the central ASU PSF and the average of the four DM spots was calculated within each wavelength slice of each ND image, and similarly for the average of the four DM spots and four satellite spots (or four second-order satellite spots) in each wavelength slice of each coronagraphic image.

To estimate the flux ratio we minimized the following objective function using a Nelder-Mead downhill simplex algorithm

$$
f(a, b)=\sum_{r_{i}<c}\left[10^{a} X_{i}-\left(Y_{i}-b\right)\right]^{2}
$$

where $X$ is the brighter source (the ASU in the ND images or the average of the four DM spots in the coronagraphic images), $Y$ is the fainter source, $a$ is a scaling factor (logarithmic to enforce positive values), and $b$ is a DC offset term to account for imperfect background subtraction. The summation is computed over all pixels $i$, where the radius $r_{i}$ is less than the fitting radius $c$. This process was repeated for a number of different fitting radii (0.8-3 $\lambda / D)$, high-pass filter sizes (including no high-pass filter), with and without the DC offset term, and with and without a mask on the central pixel to account for potential non-linearity and/or saturation. For most of the data taken in 2017, the phase of the sine wave on the DM was modulated to account for non-common path aberrations causing speckles to interfere with the faint DM spots, as discussed previously. Images from the four phase angles were averaged together before performing the PSF fitting.

The satellite spot ratio for each wavelength slice was computed by performing this PSF fitting analysis on a temporally-averaged data cube, while the uncertainties were estimated by taking the standard deviation of the ratios calculated from the individual images, normalized by the square root of the number of images. The final satellite spot ratio was then calculated by taking the average of the ratios computed in each wavelength slice where the DM spots were detected with a signal-to-noise ratio of $>10$.

\subsection{Revised photometric calibration}

The adopted satellite spot flux ratios for the modes tested between 2016 and 2017 are given in Table 1. We used a fitting radius of $1.5 \lambda / D$ and a high-pass filter size of $9.1 \lambda / D$ for each filter/apodizer combination. No new measurements of the satellite spot ratio with the K2 filter were performed given the prohibitively low throughput of the ASU fibre at these wavelengths. This re-analysis yielded a $\sim 15 \%$ lower satellite spot flux ratio at $H$ than previous analyses ${ }^{17}$ the most commonly used filter for integral field spectroscopy observations. A subset of the 
Table 1. Satellite spot flux ratios

\begin{tabular}{|c|c|c|c|c|}
\hline Filter & Apodizer & Order & Ratio $\left(\times 10^{-4}\right)$ & $\Delta m(\mathrm{mag})$ \\
\hline Y & Y & 1 & $1.60 \pm 0.09$ & $9.49 \pm 0.06$ \\
Y & Y & 2 & $1.42 \pm 0.12$ & $9.62 \pm 0.10$ \\
Y & H & 1 & $1.71 \pm 0.15$ & $9.42 \pm 0.10$ \\
Y & H & 2 & $1.37 \pm 0.11$ & $9.66 \pm 0.09$ \\
\hline J & J & 1 & $1.84 \pm 0.08$ & $9.34 \pm 0.05$ \\
J & J & 2 & $1.47 \pm 0.08$ & $9.66 \pm 0.06$ \\
J & H & 1 & $1.92 \pm 0.07$ & $9.29 \pm 0.04$ \\
J & H & 2 & $1.41 \pm 0.08$ & $9.62 \pm 0.06$ \\
\hline H & H & 1 & $1.74 \pm 0.03$ & $9.40 \pm 0.02$ \\
\hline K1 & K1 & 1 & $2.12 \pm 0.03$ & $9.19 \pm 0.01$ \\
K1 & H & 1 & $1.80 \pm 0.04$ & $9.36 \pm 0.02$ \\
\hline
\end{tabular}

filter and apodizer combinations $(\mathrm{Y} / \mathrm{Y}, \mathrm{H} / \mathrm{H})$ were repeated several months later using the same experimental setup, yielding flux ratios consistent with those presented in Table 1.

\section{ASTROMETRIC CALIBRATION}

\subsection{Overview}

Interpretation of observations obtained with GPI rely on a precise and accurate astrometric calibration of the instrument in order to convert pixel offsets between the position of the star (measured using the satellite spots) and the position of the faint companion into a sky-plane separation and position angle. This conversion is necessary for rejecting non-associated background stars, ${ }^{21}$ and for monitoring the orbits of planetary-mass companions, ${ }^{22}$ both of which can rely on data obtained from multiple instruments each with their own astrometric calibration. The previous astrometric calibration of $\mathrm{GPI}^{23}$ was based on observations obtained during the commissioning of the instrument in 2013 and 2014. Since this analysis was completed, several issues with the pipeline that have affected these astrometric measurements have been identified and fixed. ${ }^{24}$ These various issues all contributed to a significant error in the determination of the parallactic angle at the exposure mid-point, critical for determining the angle of North within each GPI image as it is mounted on the Cassegrain port of Gemini South with the image derotator disabled (Fig. 4). Here we give a brief summary of these various pipeline fixes and report the revised astrometric calibration of the instrument derived from a re-analysis of observations obtained during commissioning, and an analysis of data obtained afterwards during routine science operations.

\subsection{Pipeline fixes}

\subsubsection{Exposure start and stop times}

Precise exposure start and end times are required in order to compute the average parallactic angle of an observation. The previous version of the GPI DRP was not accurately calculating the exposure start time for observations that consisted of more than one coadds. The header contains several timestamps, in two different time scales, of various events during the acquisition of an image with the IFS. The pipeline used one of these keywords, UTEND, that is written momentarily after the last pixel of the detector is read to signify the end of an exposure. The effective exposure stop time is therefore half a read time before UTEND, when half of the pixels have been read out. As there was no timestamp written immediately prior to an integration starting due to the continuous reset mode of the detector, the exposure start time was calculated backwards from UTEND and the number of coadds and number of reads per coadd. Unfortunately, the previous version of the pipeline used an erroneous formula to compute the duration of an exposure for images with $n_{\text {coadd }}>1$, and thus calculated an incorrect exposure start time. This effect is most pronounced for images with short exposure times (low $\left.n_{\text {read }}\right)$ and large $n_{\text {coadd }}$. As an example, the error on the exposure start time for an exposure with $n_{\text {read }}=2$ and $n_{\text {coadd }}=10$ would be approximately $40 \mathrm{~s}$, an error equivalent to $98 \%$ of the duration of the exposure. A significant number of the astrometric calibration observations were taken using coadds, and those observed at 


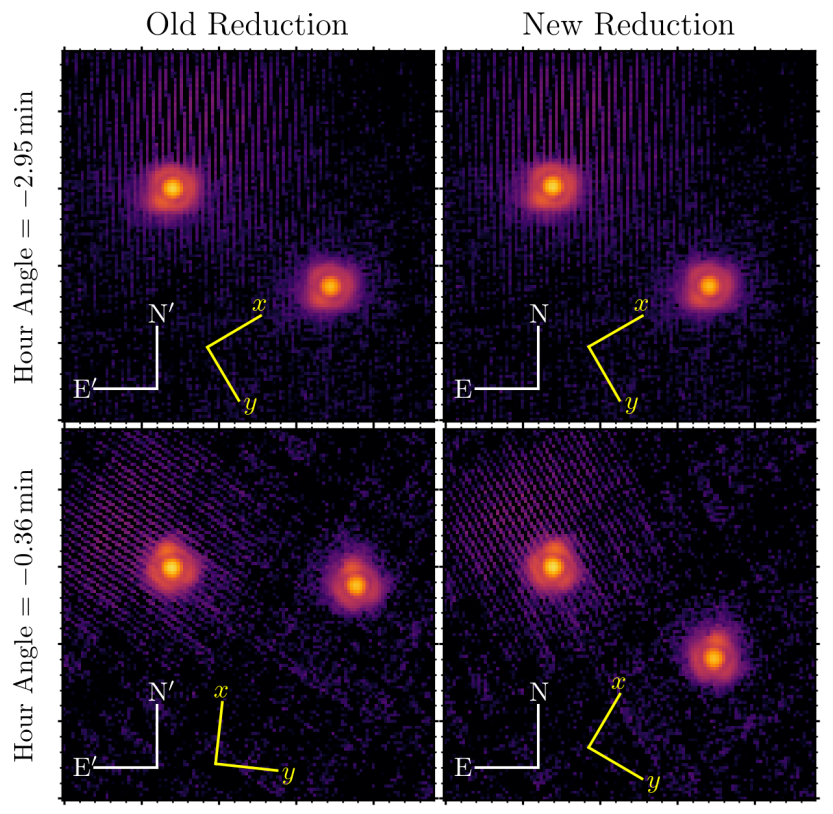

Figure 4. Two GPI images of the binary star HD 6307 demonstrating the error in the calculation of the average parallactic angle in the previous version of the pipeline. The two columns show reductions with the previous (left) and current (right) version of the pipeline, while the two rows show the binary star a few minutes (top) and a few seconds (bottom) before meridian passage. The coordinate axes of the sky (white) and detector (yellow) are shown. A significant change in the position angle of the companion is seen for data reduced using the previous version of the pipeline, whereas the position angle is stable as a function of hour angle with the current version.

a small zenith distance where the parallactic angle is changing rapidly were most affected. This error has been fixed in the latest version of the pipeline.

\subsubsection{Average parallactic angle}

The previous version of the pipeline contained an error when computing the average parallactic angle of an exposure using Romberg's method. The parallactic angle as a function of hour angle of a northern target exhibits a discontinuity as the target passes through the meridian. This discontinuity was avoided by applying Romberg's method to the parallactic angle curve on either side of meridian passage as

$$
p_{\text {avg }}=\frac{1}{H_{1}-H_{0}}\left[\int_{H_{0}}^{0}[p(H, \phi, \delta)+2 \pi] d H+\int_{0}^{H_{1}} p(H, \phi, \delta) d H\right] .
$$

where $p_{\text {avg }}$ is the average parallactic angle, $H_{0}$ and $H_{1}$ are the hour angles at the start and end of the integration, $\phi$ is the observatory latitude, and $\delta$ the declination of the target. The previous version of the pipeline contained an error in this calculation that did not properly account for this discontinuity. This error only affected observations of northern targets that transited the meridian between the start and end of an exposure. This error has been fixed in the latest version of the pipeline.

\subsubsection{Instrument clock drift}

The clocks on the various computers within GPI are regularly synchronized with a time server maintained by the Gemini Observatory to minimize the drift between these computers and UTC. After an exhaustive search of GPI observations obtained between 2014 and 2019, we identified several periods of time where the clock on the GPI IFS computer had drifted significantly with respect to UTC. The time on the IFS computer is used for the various timestamps saved within the FITS header that are used to compute the exposure start and end 
Table 2. GPI north offset angle

\begin{tabular}{|c|c|c|}
\hline Date start & Date end & $\theta_{\text {truth }}-\theta_{\text {GPI }}$ \\
\hline $2013-11-11$ & $2014-09-08$ & $0.23 \pm 0.11 \mathrm{deg}$ \\
$2014-09-08$ & $2015-10-31$ & $0.17 \pm 0.14 \mathrm{deg}$ \\
$2015-10-31$ & $2016-09-05$ & $0.21 \pm 0.23 \mathrm{deg}$ \\
$2016-09-05$ & $2017-10-13$ & $0.32 \pm 0.15 \mathrm{deg}$ \\
$2017-10-13$ & $2018-09-01$ & $0.28 \pm 0.19 \mathrm{deg}$ \\
$2018-09-01$ & $2019-08-27$ & $0.45 \pm 0.11 \mathrm{deg}$ \\
\hline
\end{tabular}

times and the average parallactic angle of the exposure (see Section 3.2.1). While one period can be attributed to a mis-configuration of the Gemini time server, the cause of the remaining periods of clock drift is not fully understood. The start of the clock drifts appear correlated with restarts of the IFS computer. One hypothesis is that the restart of the IFS causes the time to be set according to the BIOS until the regular synchronization with the Gemini time server. This can be up to a week later depending on when the restart occurred. We have constructed a model describing the offset between the IFS clock and UTC that is used to correct the timestamps within the FITS headers. This correction is applied automatically in the latest version of the pipeline during the periods identified to have a significant clock drift.

\subsubsection{Cassegrain de-rotator}

GPI exclusively operates in angular differential imaging mode where the instrument pupil is (in theory) fixed relative to the telescope pupil, and the angle of north varies with the parallactic angle of the target being observed. For an instrument mounted on the Cassegrain port of an ideal alt-az telescope, this observing mode can be achieved by disabling the Cassegrain instrument rotator that is normally used to maintain a fixed position angle between the angle of north and the columns on the detector. With the drive disabled, the angle between vertical (topocentric elevation, not telescope elevation) and the columns on the detector is instead constant, with the angle of north varying. As no telescope is perfectly constructed, the instrument rotator is needed to compensate for the telescope non-perpendicularity in order to keep the vertical angle fixed relative to the columns of the GPI IFS. Due to difficulties in keeping the AO loop closed for targets passing through the meridian with very small zenith distances it became common practice to keep the instrument rotator drive disabled while GPI was in operation. Unfortunately, this causes a small but detectable shift in the vertical angle for stars observed with very small zenith distances, leading to an erroneous north angle for these observations. This can be as high as 0.5 degrees for stars with zenith distances of a few degrees. Using the GPIES database ${ }^{25}$ we constructed a model of the non-perpendicularity of the Gemini South telescope using instrument rotator position angle data saved in the header of GPI observations taken while the instrument rotator was enabled. A lookup table was constructed using this model that is used by the DRP to automatically apply a correction to an observation based upon their unique Gemini identifier (DATALAB). No correction is applied for observations not within this table, although it covers almost all of the period in which the instrument was in regular operation.

\subsection{Revised astrometric calibration}

The various fixes to the pipeline described in Section 3.2 motivated a re-analysis of observations of calibration binaries used to define the initial astrometric calibration of the instrument, ${ }^{23}$ augmented with observations of the same and other binaries observed between 2014 and 2019. The GPI observations were reduced in a consistent fashion using version 1.5 of the DRP that incorporated all of the changes described previously. We compared the measured detector separation and position angles to ephemerides of these binary systems derived from orbital fits to Keck/NIRC2 observations, an instrument that has an extremely precise astrometric calibration linked to globular clusters and galactic center masers. ${ }^{26,27}$ Based on these comparisons, we derived a plate scale for the GPI IFS of $14.161 \pm 0.021 \mathrm{mas} \mathrm{px}^{-1}$, consistent with the previous value of $14.166 \pm 0.007 \mathrm{mas} \mathrm{px}^{-1}$ albeit with a larger uncertainty. We also find evidence of a time-variable north angle offset $\left(\theta_{\text {truth }}-\theta_{\mathrm{GPI}}\right)$, given in Table 2. The cause of this variation is not known; some rotations internal to the instrument were excluded by the stability of the angle between the four satellite spots within the regular alignment images taken at the 
start of each night. The limiting magnitude of GPI $(I<10)$ prevented us from using dense globular clusters as astrometric calibration fields. These are far better suited for confirming the stability of the north angle, as relative motion between the stars within the cluster is expected to be small over the duration of the instrument lifetime, whereas the binaries used in this analysis had periods short enough that orbital motion could be detected over several months given the precision of the observations.

\section{INSTRUMENTAL POLARIZATION SUBTRACTION}

A new step to suppress instrumental polarization noise in GPI polarimetric images of circumstellar debris disks has been added at the end of the standard reduction process via a standalone Python script. That standard process has already been described in several papers..$^{9,13,28}$ In brief, it starts from raw images in which light from the astrophysical scene is divided into two orthogonal polarization states that are modulated by four rotations of a half-wave plate throughout the observing sequence. We then extract Stokes vectors from combinations of those individual frames to produce the final data products of datacubes containing images for Stokes vectors $\mathcal{I}, \mathcal{Q}, \mathcal{U}, \mathcal{Q}_{\phi}$, and $\mathcal{U}_{\phi}$, where the latter two are the radial components of the traditional $\mathcal{Q}$ and $\mathcal{U}$ vectors. In an intermediate step, the mean stellar polarization (a term that includes both the intrinsic polarization of the host star and the instrumental polarization) is measured in an annulus near the focal plane mask edge and then subtracted off from each pixel after scaling by that pixel's total intensity.

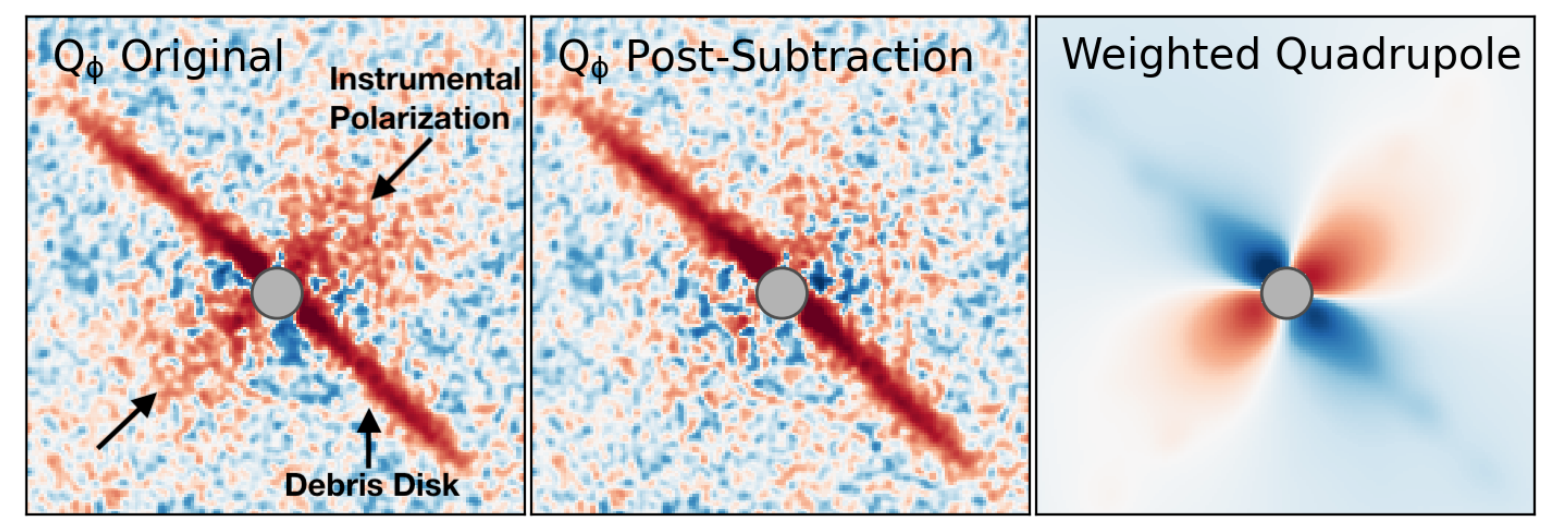

Figure 5. Example results of the instrumental polarization subtraction for a debris disk (HD 32297), shown on a log scale with negative values in blue. Left: Original $\mathcal{Q}_{\phi, i}$ image (smoothed), with the positive components of the instrumental polarization roughly perpendicular to the disk and the negative components overlapping the disk (but easily seen in $\mathcal{U}_{\phi, i}$ ). Middle: $\mathcal{Q}_{\phi, i}$ image after subtraction of the best-fit weighted quadrupole function. Right: Weighted quadrupole function, rotated from the best-fit function in $\mathcal{U}_{\phi, i}$. The gray circle marks the region occulted by GPI's focal plane mask.

It became clear partway through the GPIES debris disk survey that a quadrupole-like pattern occasionally remained in the $\mathcal{Q}_{\phi}$ and $\mathcal{U}_{\phi}$ images due to imperfect subtraction of the instrumental polarization. Our mitigation approach has been to fit and subtract this quadrupole term from the $\mathcal{Q}_{\phi}$ and $\mathcal{U}_{\phi}$ images. The current iteration of this method involves first creating a set of intermediate radial Stokes datacubes for each data set, where each cube is created from a subset of four individual polarimetric frames, which is the minimum needed to retrieve the full set of Stokes vectors (occasionally the last datacube will include up to seven frames depending on the number of images in the data set). For each intermediate datacube $i$, we fit a function of the form $B_{i}=W_{i} \sin 2\left(\theta+\theta_{i}\right)$ to the $\mathcal{U}_{\phi, i}$ image by varying a weighting function $W_{i}$ and offset rotation angle $\theta_{i}$ to minimize the sum of the squared residuals. $W_{i}$ is a linear combination of $\mathcal{I}_{i}$ (the total intensity image) and the radial profile of $\mathcal{U}_{\phi, i}$ (where the value at each radius from the star is the azimuthal median). We fit to the $\mathcal{U}_{\phi}$ vector because it is assumed to contain only noise (i.e. no disk signal), as single scatterings of photons from optically thin debris disks are expected to produce only $\mathcal{Q}_{\phi}$ polarization orientations. For bright debris disks that have $\mathcal{I}_{i}$ brightnesses comparable to the stellar PSF brightness, this weighting approach can potentially "self-subtract" that disk brightness from $\mathcal{Q}_{\phi, i}$; however, we qualitatively find this effect to be small and so far consider it negligible compared to our overall photometric uncertainties on extended emission (typically $\gtrsim 10 \%$ ). The best-fit function $B_{i}$ is subtracted from 
the $\mathcal{U}_{\phi, i}$ image and also rotated $45^{\circ}$ counterclockwise and subtracted from the $\mathcal{Q}_{\phi, i}$ image (Fig. 5). We then average together all subtracted intermediate datacubes to produce final instrumental-polarization subtracted $\mathcal{Q}_{\phi}$ and $\mathcal{U}_{\phi}$ images for each data set.

We have found that this approach significantly reduces both low and high frequency systematic noise related to the instrumental polarization. Additionally, this current method is an incremental improvement over our previous method, which simply fit a weighted quadrupole function to a data set's final $\mathcal{U}_{\phi}$ image, and subtracted it from $\mathcal{U}_{\phi}$ and then $\mathcal{Q}_{\phi}$ after a $45^{\circ}$ rotation. ${ }^{13}$ Fitting for the instrumental polarization noise several times across the data set, rather than just once in aggregate, better accounts for changes in atmospheric seeing, AO correction, star centering behind the focal plane mask, parallactic rotation, and other time variable factors that affect the instrumental polarization signal.

\section{ACKNOWLEDGMENTS}

The authors were supported in part by NSF AST-1411868, AST-1518332, NASA NNX14AJ80G, NNX15AC89G, NNX15AD95G, NSSC17K0535, NNG16PJ24C, and NASA 80NSSC17K0535. J.R. benefited from Fonds de Recherche du Québec and is supported by the French National Research Agency in the framework of the Investissements d'Avenir program (ANR-15-IDEX-02), through the funding of the "Origin of Life" project of the Univ. Grenoble-Alpes. J.J.W. is supported by the Heising-Simons Foundation 51 Pegasi b postdoctoral fellowship. This work benefited from NASA's Nexus for Exoplanet System Science (NExSS) research coordination network sponsored by NASA's Science Mission Directorate. Based on observations obtained at the international Gemini Observatory, a program of NSF's NOIRLab, which is managed by the Association of Universities for Research in Astronomy (AURA) under a cooperative agreement with the National Science Foundation. on behalf of the Gemini Observatory partnership: the National Science Foundation (United States), National Research Council (Canada), Agencia Nacional de Investigación y Desarrollo (Chile), Ministerio de Ciencia, Tecnología e Innovación (Argentina), Ministério da Ciência, Tecnologia, Inovações e Comunicações (Brazil), and Korea Astronomy and Space Science Institute (Republic of Korea).

\section{REFERENCES}

[1] Macintosh, B., Graham, J. R., Ingraham, P., Konopacky, Q., Marois, C., Perrin, M., Poyneer, L., Bauman, B., Barman, T., Burrows, A. S., Cardwell, A., Chilcote, J., De Rosa, R. J., Dillon, D., Doyon, R., Dunn, J., Erikson, D., Fitzgerald, M. P., Gavel, D., Goodsell, S., Hartung, M., Hibon, P., Kalas, P., Larkin, J., Maire, J., Marchis, F., Marley, M. S., McBride, J., Millar-Blanchaer, M., Morzinski, K., Norton, A., Oppenheimer, B. R., Palmer, D., Patience, J., Pueyo, L., Rantakyro, F., Sadakuni, N., Saddlemyer, L., Savransky, D., Serio, A., Soummer, R., Sivaramakrishnan, A., Song, I., Thomas, S., Wallace, J. K., Wiktorowicz, S., and Wolff, S., "First light of the Gemini Planet Imager," PNAS 111, 12661-12666 (Sept. 2014).

[2] Poyneer, L. A. and Dillon, D., "MEMS adaptive optics for the Gemini Planet Imager: control methods and validation," Ground-based and Airborne Instrumentation for Astronomy VI 6888, 68880H (Feb. 2008).

[3] Soummer, R., Sivaramakrishnan, A., Pueyo, L., Macintosh, B., and Oppenheimer, B. R., "Apodized Pupil Lyot Coronagraphs For Arbitrary Apertures. III. Quasi-Achromatic Solutions," Astrophys. J. 729, 144 (Feb. 2011).

[4] Poyneer, L. A., De Rosa, R. J., Macintosh, B., Palmer, D. W., Perrin, M. D., Sadakuni, N., Savransky, D., Bauman, B., Cardwell, A., Chilcote, J. K., Dillon, D., Gavel, D., Goodsell, S. J., Hartung, M., Hibon, P., Rantakyrö, F. T., Thomas, S., and Veran, J.-P., "On-sky performance during verification and commissioning of the Gemini Planet Imager's adaptive optics system," in [SPIE Astronomical Telescopes + Instrumentation], Marchetti, E., Close, L. M., and Veran, J.-P., eds., 91480K, SPIE (July 2014).

[5] Poyneer, L. A., Palmer, D. W., Macintosh, B., Savransky, D., Sadakuni, N., Thomas, S., Veran, J.-P., Follette, K. B., Greenbaum, A. Z., Mark Ammons, S., Bailey, V. P., Bauman, B., Cardwell, A., Dillon, D., Gavel, D., Hartung, M., Hibon, P., Perrin, M. D., Rantakyrö, F. T., Sivaramakrishnan, A., and Wang, J. J., "Performance of the Gemini Planet Imager's adaptive optics system," Appl. Opt. 55(2), 323 (2016). 
[6] Bailey, V. P., Poyneer, L. A., Macintosh, B. A., Savransky, D., Wang, J. J., De Rosa, R. J., Follette, K. B., Ammons, S. M., Hayward, T., Ingraham, P., Maire, J., Palmer, D. W., Perrin, M. D., Rajan, A., Rantakyrö, F. T., Thomas, S., and Veran, J.-P., "Status and performance of the Gemini Planet Imager adaptive optics system," in [SPIE Astronomical Telescopes + Instrumentation], Marchetti, E., Close, L. M., and Veran, J.-P., eds., 99090V, SPIE (July 2016).

[7] Chilcote, J. K., Larkin, J. E., Maire, J., Perrin, M. D., Fitzgerald, M. P., Doyon, R., Thibault, Simon, Bauman, B., Macintosh, B. A., Graham, J. R., and Saddlemyer, L., "Performance of the integral field spectrograph for the Gemini Planet Imager," in [SPIE Astronomical Telescopes + Instrumentation], McLean, I. S., Ramsay, S. K., and Takami, H., eds., 84468W, SPIE (Sept. 2012).

[8] Larkin, J. E., Chilcote, J. K., Aliado, T., Bauman, B. J., Brims, G., Canfield, J. M., Cardwell, A., Dillon, D., Doyon, R., Dunn, J., Fitzgerald, M. P., Graham, J. R., Goodsell, S., Hartung, M., Hibon, P., Ingraham, P., Johnson, C. A., Kress, E., Konopacky, Q. M., Macintosh, B. A., Magnone, K. G., Maire, J., McLean, I. S., Palmer, D., Perrin, M. D., Quiroz, C., Rantakyro, F., Sadakuni, N., Saddlemyer, L., Serio, A., Thibault, Simon, Thomas, S. J., Vallee, P., and Weiss, J. L., "The integral field spectrograph for the Gemini planet imager," in [SPIE Astronomical Telescopes + Instrumentation], Ramsay, S. K., McLean, I. S., and Takami, H., eds., 91471K, SPIE (July 2014).

[9] Perrin, M. D., Duchêne, G., Millar-Blanchaer, M., Fitzgerald, M. P., Graham, J. R., Wiktorowicz, S. J., Kalas, P. G., Macintosh, B., Bauman, B., Cardwell, A., Chilcote, J., De Rosa, R. J., Dillon, D., Doyon, R., Dunn, J., Erikson, D., Gavel, D., Goodsell, S., Hartung, M., Hibon, P., Ingraham, P., Kerley, D., Konapacky, Q., Larkin, J. E., Maire, J., Marchis, F., Marois, C., Mittal, T., Morzinski, K. M., Oppenheimer, B. R., Palmer, D. W., Patience, J., Poyneer, L., Pueyo, L., Rantakyrö, F. T., Sadakuni, N., Saddlemyer, L., Savransky, D., Soummer, R., Sivaramakrishnan, A., Song, I., Thomas, S., Wallace, J. K., Wang, J. J., and Wolff, S. G., "Polarimetry with the Gemini Planet Imager: Methods, Performance at First Light, and the Circumstellar Ring around HR 4796A," Astrophys. J. 799, 182 (Feb. 2015).

[10] Macintosh, B., Graham, J. R., Barman, T., De Rosa, R. J., Konopacky, Q., Marley, M. S., Marois, C., Nielsen, E. L., Pueyo, L., Rajan, A., Rameau, J., Saumon, D., Wang, J. J., Patience, J., Ammons, M., Arriaga, P., Artigau, E., Beckwith, S., Brewster, J., Bruzzone, S., Bulger, J., Burningham, B., Burrows, A. S., Chen, C., Chiang, E., Chilcote, J. K., Dawson, R. I., Dong, R., Doyon, R., Draper, Z. H., Duchêne, G., Esposito, T. M., Fabrycky, D., Fitzgerald, M. P., Follette, K. B., Fortney, J. J., Gerard, B., Goodsell, S., Greenbaum, A. Z., Hibon, P., Hinkley, S., Cotten, T. H., Hung, L. W., Ingraham, P., Johnson-Groh, M., Kalas, P., Lafrenière, D., Larkin, J. E., Lee, J., Line, M., Long, D., Maire, J., Marchis, F., Matthews, B. C., Max, C. E., Metchev, S., Millar-Blanchaer, M. A., Mittal, T., Morley, C. V., Morzinski, K. M., Murray-Clay, R., Oppenheimer, R., Palmer, D. W., Patel, R., Perrin, M. D., Poyneer, L. A., Rafikov, R. R., Rantakyrö, F. T., Rice, E. L., Rojo, P., Rudy, A. R., Ruffio, J. B., Ruiz, M. T., Sadakuni, N., Saddlemyer, L., Salama, M., Savransky, D., Schneider, A. C., Sivaramakrishnan, A., Song, I., Soummer, R., Thomas, S., Vasisht, G., Wallace, J. K., Ward-Duong, K., Wiktorowicz, S. J., Wolff, S. G., and Zuckerman, B., "Discovery and spectroscopy of the young jovian planet 51 Eri b with the Gemini Planet Imager," Science 350, 64-67 (Oct. 2015).

[11] De Rosa, R. J., Nielsen, E. L., Blunt, S. C., Graham, J. R., Konopacky, Q. M., Marois, C., Pueyo, L., Rameau, J., Ryan, D. M., Wang, J. J., Bailey, V., Chontos, A., Fabrycky, D. C., Follette, K. B., Macintosh, B., Marchis, F., Ammons, S. M., Arriaga, P., Chilcote, J. K., Cotten, T. H., Doyon, R., Duchêne, G., Esposito, T. M., Fitzgerald, M. P., Gerard, B., Goodsell, S. J., Greenbaum, A. Z., Hibon, P., Ingraham, P., Johnson-Groh, M., Kalas, P. G., Lafrenière, D., Maire, J., Metchev, S., Millar-Blanchaer, M. A., Morzinski, K. M., Oppenheimer, R., Patel, R. I., Patience, J. L., Perrin, M. D., Rajan, A., Rantakyrö, F. T., Ruffio, J.-B., Schneider, A. C., Sivaramakrishnan, A., Song, I., Tran, D., Vasisht, G., Ward-Duong, K., and Wolff, S. G., "Astrometric Confirmation and Preliminary Orbital Parameters of the Young Exoplanet 51 Eridani b with the Gemini Planet Imager," ApJL 814, L3 (Nov. 2015).

[12] Nielsen, E. L., De Rosa, R. J., Macintosh, B., Wang, J. J., Ruffio, J.-B., Chiang, E., Marley, M. S., Saumon, D., Savransky, D., Ammons, S. M., Bailey, V. P., Barman, T., Blain, C., Bulger, J., Burrows, A., Chilcote, J., Cotten, T., Czekala, I., Doyon, R., Duchêne, G., Esposito, T. M., Fabrycky, D., Fitzgerald, M. P., Follette, K. B., Fortney, J. J., Gerard, B. L., Goodsell, S. J., Graham, J. R., Greenbaum, A. Z., Hibon, P., Hinkley, S., Hirsch, L. A., Hom, J., Hung, L.-W., Dawson, R. I., Ingraham, P., Kalas, P., Konopacky, 
Q., Larkin, J. E., Lee, E. J., Lin, J. W., Maire, J., Marchis, F., Marois, C., Metchev, S., Millar-Blanchaer, M. A., Morzinski, K. M., Oppenheimer, R., Palmer, D., Patience, J., Perrin, M., Poyneer, L., Pueyo, L., Rafikov, R. R., Rajan, A., Rameau, J., Rantakyrö, F. T., Ren, B., Schneider, A. C., Sivaramakrishnan, A., Song, I., Soummer, R., Tallis, M., Thomas, S., Ward-Duong, K., and Wolff, S., "The Gemini Planet Imager Exoplanet Survey: Giant Planet and Brown Dwarf Demographics from 10 to 100 au," AJ 158, 13 (July 2019).

[13] Esposito, T. M., Kalas, P., Fitzgerald, M. P., Millar-Blanchaer, M. A., Duchêne, G., Patience, J., Hom, J., Perrin, M. D., De Rosa, R. J., Chiang, E., Czekala, I., Macintosh, B., Graham, J. R., Ansdell, M., Arriaga, P., Bruzzone, S., Bulger, J., Chen, C. H., Cotten, T., Dong, R., Draper, Z. H., Follette, K. B., Hung, L.-W., Lopez, R., Matthews, B. C., Mazoyer, J., Metchev, S., Rameau, J., Ren, B., Rice, M., Song, I., Stahl, K., Wang, J., Wolff, S., Zuckerman, B., Ammons, S. M., Bailey, V. P., Barman, T., Chilcote, J., Doyon, R., Gerard, B. L., Goodsell, S. J., Greenbaum, A. Z., Hibon, P., Hinkley, S., Ingraham, P., Konopacky, Q., Maire, J., Marchis, F., Marley, M. S., Marois, C., Nielsen, E. L., Oppenheimer, R., Palmer, D., Poyneer, L., Pueyo, L., Rajan, A., Rantakyrö, F. T., Ruffio, J.-B., Savransky, D., Schneider, A. C., Sivaramakrishnan, A., Soummer, R., Thomas, S., and Ward-Duong, K., "Debris Disk Results from the Gemini Planet Imager Exoplanet Survey's Polarimetric Imaging Campaign," AJ 160, 24 (July 2020).

[14] Perrin, M. D., Maire, J., Ingraham, P., Savransky, D., Millar-Blanchaer, M., Wolff, S. G., Ruffio, J.-B., Wang, J. J., Draper, Z. H., Sadakuni, N., Marois, C., Rajan, A., Fitzgerald, M. P., Macintosh, B., Graham, J. R., Doyon, R., Larkin, J. E., Chilcote, J. K., Goodsell, S. J., Palmer, D. W., Labrie, K., Beaulieu, M., De Rosa, R. J., Greenbaum, A. Z., Hartung, M., Hibon, P., Konopacky, Q., Lafrenière, D., Lavigne, J.-F., Marchis, F., Patience, J., Pueyo, L., Rantakyrö, F. T., Soummer, R., Sivaramakrishnan, A., Thomas, S., Ward-Duong, K., and Wiktorowicz, S., "Gemini Planet Imager observational calibrations I: Overview of the GPI data reduction pipeline," Proc. SPIE 9147, 91473J-91473J-13 (July 2014).

[15] Perrin, M. D., Soummer, R., Elliott, E. M., Lallo, M. D., and Sivaramakrishnan, A., "Simulating point spread functions for the James Webb Space Telescope with WebbPSF," in [SPIE Astronomical Telescopes + Instrumentation], Clampin, M. C., Fazio, G. G., MacEwen, H. A., and Oschmann, J. M., eds., 84423D, SPIE (Sept. 2012).

[16] Beuzit, J. L., Vigan, A., Mouillet, D., Dohlen, K., Gratton, R., Boccaletti, A., Sauvage, J. F., Schmid, H. M., Langlois, M., Petit, C., Baruffolo, A., Feldt, M., Milli, J., Wahhaj, Z., Abe, L., Anselmi, U., Antichi, J., Barette, R., Baudrand, J., Baudoz, P., Bazzon, A., Bernardi, P., Blanchard, P., Brast, R., Bruno, P., Buey, T., Carbillet, M., Carle, M., Cascone, E., Chapron, F., Charton, J., Chauvin, G., Claudi, R., Costille, A., De Caprio, V., de Boer, J., Delboulbe, A., Desidera, S., Dominik, C., Downing, M., Dupuis, O., Fabron, C., Fantinel, D., Farisato, G., Feautrier, P., Fedrigo, E., Fusco, T., Gigan, P., Ginski, C., Girard, J., Giro, E., Gisler, D., Gluck, L., Gry, C., Henning, T., Hubin, N., Hugot, E., Incorvaia, S., Jaquet, M., Kasper, M., Lagadec, E., Lagrange, A.-M., Le Coroller, H., Le Mignant, D., Le Ruyet, B., Lessio, G., Lizon, J. L., Llored, M., Lundin, L., Madec, F., Magnard, Y., Marteaud, M., Martinez, P., Maurel, D., Menard, F., Mesa, D., Möller-Nilsson, O., Moulin, T., Moutou, C., Origné, A., Parisot, J., Pavlov, A., Perret, D., Pragt, J., Puget, P., Rabou, P., Ramos, J., Reess, J. M., Rigal, F., Rochat, S., Roelfsema, R., Rousset, G., Roux, A., Saisse, M., Salasnich, B., Santambrogio, E., Scuderi, S., Segransan, D., Sevin, A., Siebenmorgen, R., Soenke, C., Stadler, E., Suarez, M., Tiphène, D., Turatto, M., Udry, S., Vakili, F., Waters, L. B. F. M., Weber, L., Wildi, F., Zins, G., and Zurlo, A., "SPHERE: the exoplanet imager for the Very Large Telescope," $A \& 3 A$ 631, A155 (Nov. 2019).

[17] Maire, J., Ingraham, P. J., De Rosa, R. J., Perrin, M. D., Rajan, A., Savransky, D., Wang, J. J., Ruffio, J.-B., Wolff, S. G., Chilcote, J. K., Doyon, R., Graham, J. R., Greenbaum, A. Z., Konopacky, Q. M., Larkin, J. E., Macintosh, B. A., Marois, C., Millar-Blanchaer, M., Patience, J., Pueyo, L. A., Sivaramakrishnan, A., Thomas, S. J., and Weiss, J. L., "Gemini planet imager observational calibrations VI: photometric and spectroscopic calibration for the integral field spectrograph," Proc. SPIE 9147, 85-914785-15 (July 2014).

[18] Wang, J. J., Rajan, A., Graham, J. R., Savransky, D., Ingraham, P. J., Ward-Duong, K., Patience, J., De Rosa, R. J., Bulger, J., Sivaramakrishnan, A., Perrin, M. D., Thomas, S. J., Sadakuni, N., Greenbaum, A. Z., Pueyo, L., Marois, C., Oppenheimer, B. R., Kalas, P., Cardwell, A., Goodsell, S., Hibon, P., and Rantakyrö, F. T., "Gemini planet imager observational calibrations VIII: characterization and role of satellite spots," Proc. SPIE 9147, 55-914755-15 (July 2014). 
[19] Sivaramakrishnan, A. and Oppenheimer, B. R., "Astrometry and Photometry with Coronagraphs," Astrophys. J. 647, 620-629 (Aug. 2006).

[20] Wang, J. J., Perrin, M. D., Savransky, D., Arriaga, P., Chilcote, J. K., De Rosa, R. J., Millar-Blanchaer, M. A., Shapiro, J., Rameau, J., Wolff, S. G., Graham, J. R., Macintosh, B. A., Marois, C., and Ruffio, J.-B., "The automated data processing architecture for the GPI Exoplanet Survey," in [Techniques and Instrumentation for Detection of Exoplanets VIII], Shaklan, S., ed., 78, SPIE (Sept. 2017).

[21] Nielsen, E. L., De Rosa, R. J., Rameau, J., Wang, J. J., Esposito, T. M., Millar-Blanchaer, M. A., Marois, C., Vigan, A., Ammons, S. M., Artigau, É., Bailey, V. P., Blunt, S., Bulger, J., Chilcote, J., Cotten, T., Doyon, R., Duchêne, G., Fabrycky, D., Fitzgerald, M. P., Follette, K. B., Gerard, B. L., Goodsell, S. J., Graham, J. R., Greenbaum, A. Z., Hibon, P., Hinkley, S., Hung, L.-W., Ingraham, P., Jensen-Clem, R., Kalas, P., Konopacky, Q., Larkin, J. E., Macintosh, B., Maire, J., Marchis, F., Metchev, S., Morzinski, K. M., MurrayClay, R. A., Oppenheimer, R., Palmer, D., Patience, J., Perrin, M., Poyneer, L., Pueyo, L., Rafikov, R. R., Rajan, A., Rantakyrö, F. T., Ruffio, J.-B., Savransky, D., Schneider, A. C., Sivaramakrishnan, A., Song, I., Soummer, R., Thomas, S., Wallace, J. K., Ward-Duong, K., Wiktorowicz, S., and Wolff, S., "Evidence That the Directly Imaged Planet HD 131399 Ab Is a Background Star," AJ 154, 218 (Dec. 2017).

[22] De Rosa, R. J., Nielsen, E. L., Wang, J. J., Ammons, S. M., Duchêne, G., Macintosh, B., Rameau, J., Bailey, V. P., Barman, T., Bulger, J., Chilcote, J., Cotten, T., Doyon, R., Esposito, T. M., Fitzgerald, M. P., Follette, K. B., Gerard, B. L., Goodsell, S. J., Graham, J. R., Greenbaum, A. Z., Hibon, P., Hom, J., Hung, L.-W., Ingraham, P., Kalas, P., Konopacky, Q., Larkin, J. E., Maire, J., Marchis, F., Marley, M. S., Marois, C., Metchev, S., Millar-Blanchaer, M. A., Oppenheimer, R., Palmer, D., Patience, J., Perrin, M., Poyneer, L., Pueyo, L., Rajan, A., Rantakyrö, F. T., Ren, B., Ruffio, J.-B., Savransky, D., Schneider, A. C., Sivaramakrishnan, A., Song, I., Soummer, R., Tallis, M., Thomas, S., Wallace, J. K., Ward-Duong, K., Wiktorowicz, S., and Wolff, S., "An Updated Visual Orbit of the Directly Imaged Exoplanet 51 Eridani b and Prospects for a Dynamical Mass Measurement with Gaia," AJ 159, 1 (Jan. 2020).

[23] Konopacky, Q. M., Thomas, S. J., Macintosh, B. A., Dillon, D., Sadakuni, N., Maire, J., Fitzgerald, M., Hinkley, S., Kalas, P., Esposito, T., Marois, C., Ingraham, P. J., Marchis, F., Perrin, M. D., Graham, J. R., Wang, J. J., De Rosa, R. J., Morzinski, K., Pueyo, L., Chilcote, J. K., Larkin, J. E., Fabrycky, D., Goodsell, S. J., Oppenheimer, B. R., Patience, J., Saddlemyer, L., and Sivaramakrishnan, A., "Gemini planet imager observational calibrations V: astrometry and distortion," Proc. SPIE 9147, 84-914784-16 (July 2014).

[24] De Rosa, R. J., Nguyen, M. M., Chilcote, J., Macintosh, B., Perrin, M. D., Konopacky, Q., Wang, J. J., Duchêne, G., Nielsen, E. L., Rameau, J., Ammons, S. M., Bailey, V. P., Barman, T., Bulger, J., Cotten, T., Doyon, R., Esposito, T. M., Fitzgerald, M. P., Follette, K. B., Gerard, B. L., Goodsell, S. J., Graham, J. R., Greenbaum, A. Z., Hibon, P., Hung, L.-W., Ingraham, P., Kalas, P., Larkin, J. E., Maire, J., Marchis, F., Marley, M. S., Marois, C., Metchev, S., Millar-Blanchaer, M. A., Oppenheimer, R., Palmer, D., Patience, J., Poyneer, L., Pueyo, L., Rajan, A., Rantakyrö, F. T., Ruffio, J.-B., Savransky, D., Schneider, A. C., Sivaramakrishnan, A., Song, I., Soummer, R., Thomas, S., Wallace, J. K., Ward-Duong, K., Wiktorowicz, S., and Wolff, S., "Revised astrometric calibration of the Gemini Planet Imager," J. Astron. Telesc. Instrum. Syst. 6, 015006 (Jan. 2020).

[25] Savransky, D., Shapiro, J., Bailey, V. P., De Rosa, R. J., Wang, J. J., Ruffio, J.-B., Nielsen, E., Tallis, M., and Perrin, M. D., "Mining the GPIES database," in [Adaptive Optics Systems VI], Schmidt, D., Schreiber, L., and Close, L. M., eds., 17, SPIE (July 2018).

[26] Yelda, S., Lu, J. R., Ghez, A. M., Clarkson, W., Anderson, J., Do, T., and Matthews, K., "Improving Galactic Center Astrometry by Reducing the Effects of Geometric Distortion," ApJ 725, 331-352 (Dec. 2010).

[27] Service, M., Lu, J. R., Campbell, R., Sitarski, B. N., Ghez, A. M., and Anderson, J., "A New Distortion Solution for NIRC2 on the Keck II Telescope," PASP 128, 095004 (Sept. 2016).

[28] Millar-Blanchaer, M. A., Perrin, M. D., Hung, L.-W., Fitzgerald, M. P., Wang, J. J., Chilcote, J., Graham, J. R., Bruzzone, S., and Kalas, P. G., "GPI observational calibrations XIV: polarimetric contrasts and new data reduction techniques," in [Ground-based and Airborne Instrumentation for Astronomy VI], Proc. SPIE 9908, 990836 (Aug. 2016). 